\title{
Statistical properties of SZ and X-ray cluster detections
}

\author{
F. Pace ${ }^{1}$, M. Maturi ${ }^{1}$, M. Bartelmann ${ }^{1}$, N. Cappelluti ${ }^{2}$, K. Dolag $^{3}$, M. Meneghetti ${ }^{4}$, and L. Moscardini ${ }^{5,6}$ \\ ${ }^{1}$ ITA, Zentrum für Astronomie, Universität Heidelberg, Albert Überle Str. 2, 69120 Heidelberg, Germany \\ e-mail: francesco@ita.uni-heidelberg.de \\ 2 Max Planck Institut für Extraterrestrische Physik, 85478 Garching, Germany \\ 3 Max Planck Institut für Astrophysik, 85478 Garching, Germany \\ 4 INAF - Osservatorio Astronomico di Bologna, Via Ranzani 1, 40127 Bologna, Italy \\ Dipartimento di Astronomia, Università di Bologna, Via Ranzani 1, 40127 Bologna, Italy \\ ${ }^{6}$ INFN-National Institute for Nuclear Physics, Sezione di Bologna, Viale Berti Pichat 6/2, 40127 Bologna, Italy
}

Received 8 February 2008 / Accepted 29 February 2008

\section{ABSTRACT}

\begin{abstract}
Aims. We calibrate the number density, completeness, reliability, and the lower mass limit of galaxy-cluster detections through their thermal SZ signal and compare them to X-ray cluster detections.

Methods. We simulate maps of the thermal SZ effect and the X-ray emission from light cones constructed in a large, hydrodynamical, cosmological simulation volume, including realistic noise contributions. The maps are convolved with linear, optimised, single- and multi-band filters to identify local peaks and their signal-to-noise ratios. The resulting peak catalogues are then compared to the halo population in the simulation volume to identify true and spurious detections.

Results. Multi-band filtering improves the statistics of SZ cluster detections considerably compared to single-band filtering. Observations with the characteristics of ACT detect clusters with masses $M \geq 6-9 \times 10^{13} M_{\odot} / h$, quite independent of redshift, reach $50 \%$ completeness at $\sim 10^{14} M_{\odot} / h$ and $100 \%$ completeness at $\sim 2 \times 10^{14} M_{\odot} / h$. Samples are contaminated by a few spurious detections, but they are only a small percentage of all detections. This is broadly comparable to X-ray cluster detections with XMM-Newton with $100 \mathrm{ks}$ exposure time in the soft band, except that the mass limit for X-ray detections increases much more steeply with redshift than for SZ detections. A comparison of true and filtered signals in the SZ and X-ray maps confirms that the filters introduce at most a negligible bias.
\end{abstract}

Key words. cosmology: theory - cosmology: cosmic microwave background - galaxies: clusters: general $\mathrm{X}$-rays: galaxies: clusters - methods: $N$-body simulations

\section{Introduction}

Galaxy clusters efficiently trace the large-scale structure of the Universe (Eisenstein et al. 2005; Hütsi 2006), and their mass function strongly depends on the cosmological parameters (Weller \& Battye 2003; Sefusatti et al. 2007; Fang \& Haiman 2007). They can be easily studied at different frequencies, ranging from microwaves to X-rays, and via weak and strong gravitational lensing (Clowe et al. 2004; Hennawi \& Spergel 2005; Tang \& Fan 2005; Comerford et al. 2006). The Sunyaev-Zel'dovich (hereafter SZ) effect and the X-ray emission are complementary, and combining them permits individually impossible constraints. Moreover, the SZ effect allows cluster detections to larger distances than X-ray emission. In turn, gravitational lensing probes the total mass rather than the gas, and is most sensitive at intermediate distances between the sources and the observer.

Expecting wide-field data in the near future, many authors developed different techniques for detecting clusters through their thermal SZ signal (Diego et al. 2002; López-Caniego et al. 2006; Herranz et al. 2002a,b) and investigated the statistical properties of SZ detections in simulated maps (see, e.g., López-Caniego et al. 2006; Melin et al. 2006; Vale \& White 2006; Schäfer \& Bartelmann 2006). These studies were based on analytic or semi-analytic cluster models applied to cosmological simulations, but gas physics was not included, because it is known to affect the appearance of clusters (see, e.g.
Kazantzidis et al. 2004; Puchwein et al. 2005; Bonaldi et al. 2007). The study of selection functions with hydrodynamical simulations were carried out by Hallman et al. (2007). Physical and statistical properties of X-ray galaxy clusters have been extensively studied (Borgani et al. 2004; Roncarelli et al. 2006b; Mazzotta et al. 2004), showing that X-ray cluster properties are very sensitive to gas physics (see, e.g. Carlstrom et al. 2002).

In this paper, we investigate the statistical properties of cluster detections in simulated maps via single- and multi-band matched filters, applied to X-ray and thermal SZ maps. We study completeness and contamination of cluster catalogues and the lowest detectable cluster mass. We also cross-correlate X-ray and SZ cluster detections. The maps are simulated by projecting the outputs of a cosmological hydrodynamical simulation (Borgani et al. 2004) of the concordance $\Lambda$ CDM model along deep light cones. The simulation contains gravitation and gas dynamics and also includes several physical processes that affect the baryonic component, such as a star formation, supernova feedback, radiative cooling and a simple reionisation scenario.

Our final goal is to optimise cluster detections in terms of reliability and completeness with linear filtering techniques applied to multi-wavelength data. Such cluster catalogues provide information on the underlying cosmology, the statistical properties of the cluster population and its redshift evolution, and thus on the non-linear structure growth in the universe. 
We summarise the SZ effect and the X-ray emission in Sect. 2. The cosmological simulation is briefly described in Sect. 3 together with our procedure for creating SZ and X-ray maps including noise. The single and multi-band filters and our models for the SZ effect and the X-ray emission are described in Sect. 4. In Sect. 5, we analyse the properties of the synthetic catalogues obtained by applying the single- and multi-band filters, while the correlation between SZ and X-ray detections is discussed in Sect. 6. Our main results are summarised in Sect. 7.

\section{The observables}

\subsection{The SZ effect}

The SZ effect is due to the inverse Compton scattering of CMB photons off the intracluster electrons. The thermal SZ (tSZ) effect is caused by the thermal motion of the electrons, the kinetic SZ (kSZ) effect by the bulk motion of the clusters. The kSZ effect is typically an order of magnitude weaker than the tSZ effect. The tSZ effect is quantified by the dimension-less Compton $y$-parameter

$y=\frac{k_{\mathrm{B}} \sigma_{\mathrm{T}}}{m_{\mathrm{e}} c^{2}} \int \mathrm{d} \ln _{\mathrm{e}} T_{\mathrm{e}}$,

integrating over the product of the electron number density $n_{\mathrm{e}}$ and their temperature $T_{\mathrm{e}}$, where $k_{\mathrm{B}}$ is the Boltzmann constant, $\sigma_{\mathrm{T}}$ the Thompson cross-section, $m_{\mathrm{e}}$ the electron rest mass and $c$ the speed of light. The $\mathrm{tSZ}$ effect changes the CMB brightness temperature by

$\frac{\Delta T}{T}=y g_{v}(x)$

where $g_{v}(x)$ is the tSZ spectrum

$g_{v}(x)=\frac{x}{\tanh (x / 2)}-4$

as a function of the dimension-less CMB photon energy $x \equiv$ $h v /\left(k_{\mathrm{B}} T_{\mathrm{CMB}}\right)$. In the Rayleigh-Jeans limit $(x \ll 1) g_{v}(x) \simeq-2$, and the tSZ effect vanishes at $v \simeq 217 \mathrm{GHz}$.

The kSZ effect causes brightness-temperature fluctuations

$\frac{\Delta T}{T}=-b=-\frac{\sigma_{\mathrm{T}}}{c} \int \mathrm{d} \ln _{\mathrm{e}} v_{\mathrm{r}}$,

where $v_{\mathrm{r}}$ is the radial component of the cluster velocity, defined positive for receding clusters. The $\mathrm{kSZ}$ effect does not depend on frequency and is thus best estimated from observations near $v \simeq 217 \mathrm{GHz}$, where the $\mathrm{tSZ}$ effect vanishes.

\subsection{The $X$-ray emission}

X-ray emission of galaxy clusters due to thermal free-free emission (bremsstrahlung) has the bolometric emissivity

$\epsilon_{\mathrm{ff}} \approx 1.2 \times 10^{-27} T^{1 / 2} n_{\mathrm{P}}^{2} g(T)$

in cgs units, where the gas temperature $T$ is given in $\mathrm{keV}, n_{\mathrm{P}}$ is the proton density in cgs units, and $g(T)$ is the Gaunt factor. The thermal free-free emission spectrum is $f(v)=\mathrm{e}^{-h v / k_{\mathrm{B}} T} g(v, T)$, where $g(v, T)=\left(\frac{h v}{k_{\mathrm{B}} T}\right)^{-\gamma}$ and $\gamma=Z / Z_{\odot}$ is a parameter depending on the metallicity $\left(Z \approx 0.3 Z_{\odot}\right.$ ) (see e.g. Rosati et al. 2004; Hashimoto et al. 2004).
The X-ray luminosity in the energy interval $\left[E_{1}, E_{2}\right]$ is given by $L_{\text {band }}=L_{\mathrm{X}}^{\mathrm{bol}} F_{\text {band }}^{\left[E_{1}, E_{2}\right]}$, where $L_{\mathrm{X}}^{\mathrm{bol}}=\epsilon_{\mathrm{ff}} V$, and $V$ is the emitting volume. The band-function is defined as

$F_{\text {band }}^{\left[E_{1}, E_{2}\right]}(T)=\int_{x_{1,0}(1+z)}^{x_{2,0}(1+z)} C f(x) \mathrm{d} x$,

where $x$ is defined as above and $C$ is a normalisation constant defined by $C \int_{0}^{\infty} f(x) \mathrm{d} x=1$. The subscript 0 in the integration boundaries means that the band limits are taken in the observer's rest-frame, thus the $(1+z)$ term in Eq. (6) represents the K-correction. We shall consider three different bands: soft $\left[\left(E_{1}, E_{2}\right)=(0.5,2) \mathrm{keV}\right]$, hard $\left[\left(E_{1}, E_{2}\right)=(2,4) \mathrm{keV}\right]$, hardest $\left[\left(E_{1}, E_{2}\right)=(4,10) \mathrm{keV}\right]$.

\section{The simulations}

\subsection{The cosmological simulation}

To create realistic mock catalogues of SZ and X-ray halos, we use the outputs of a hydrodynamical cosmological simulation (Borgani et al. 2004) carried out using the GADGET-2 code (Springel 2005). Here we briefly report the main characteristics of the simulation. The SZ effect and X-ray properties of objects identified in this simulation are described in other studies (e.g. Diaferio et al. 2005; Roncarelli et al. 2007; Ettori et al. 2004; Murante et al. 2004; Rasia et al. 2005; Roncarelli et al. 2006a), where further detail can be found.

The simulation assumes a concordance $\Lambda \mathrm{CDM}$ model with matter density parameter $\Omega_{\mathrm{m}}=0.3$, cosmological constant $\Omega_{\Lambda}=$ 0.7 , and Hubble parameter $h \equiv\left(H_{0} / 100 \mathrm{~km} \mathrm{~s}^{-1} \mathrm{Mpc}^{-1}\right)=0.7$. The power spectrum is normalised to $\sigma_{8}=0.8$. These cosmological parameters are consistent with recent observational estimates derived from CMB and weak lensing data (e.g. Spergel et al. 2007; Hoekstra et al. 2006).

The computational box with a comoving side length of $192 \mathrm{Mpc} / \mathrm{h}$ is filled with $480^{3}$ particles each of dark matter and gas. The mass resolution is thus $4.6 \times 10^{9} M_{\odot} / h$ and $6.9 \times$ $10^{8} M_{\odot} / h$ for dark matter and gas, respectively. The Plummerequivalent gravitational softening is set to $\epsilon_{\mathrm{Pl}}=7.5 \mathrm{kpc} / \mathrm{h} \mathrm{co-}$ moving for $0 \leq z \leq 2$, and switches to physical units at higher redshifts. The gas component is treated including several physical processes: a hybrid multi-phase model for star formation in the interstellar medium (Springel \& Hernquist 2003), radiative cooling within an optically thin gas consisting of $76 \%$ of hydrogen and $24 \%$ of helium by mass, supernova feedback to model galactic outflows, and heating by a time-dependent, photoionising uniform UV background given by quasars reionising the Universe at $z \approx 6$ (Haardt \& Madau 1996). The output of the simulation consists of one hundred snapshots, logarithmically equidistant in redshift between $z=9$ and $z=0$. We use these snapshots to create our mock light cones.

\subsection{Construction of the halo catalogues}

To identify the halos in each snapshot, we run a friends-offriends algorithm with a linking length of 0.15 times the mean particle separation to identify particles belonging to the same group. Next, we identify the halo centre as the position of the particle at the local minimum of the gravitational potential. The final halo catalogue contains the positions, the virial masses, radii and redshifts of all halos in each snapshot. More details can be found in Pace et al. (2007).

Figure 1 shows the mass function of the halos contained in light cones for limiting redshifts of $z=1$ and $z=2$, averaged 


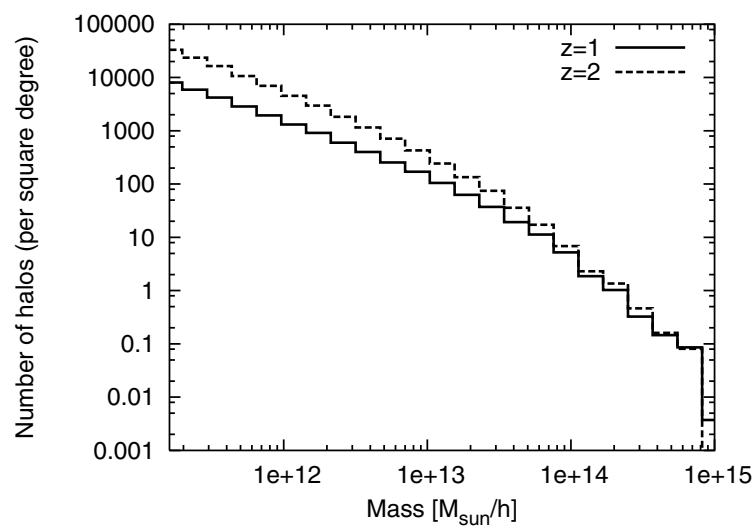

Fig. 1. Average number of halos per mass bin per square degree. The solid and dashed lines show the numbers for light cones extending to $z=1$ and $z=2$, respectively. The number of high-mass halos is approximately the same in both case, while the number of low-mass halos is higher for the deeper cone. The values are averaged over eleven different realisations.

over eleven different realisations and normalised to one square degree. Light cones reaching $z=2$ contain more low-mass halos than light cones up to $z=1$ because of their larger volume, while the number of massive halos is almost unchanged since most of them are at low redshifts.

\subsection{Construction of the simulated $S Z$ and $X$-rays light cones}

To construct a realistic three-dimensional distribution of matter, we construct several light cones by stacking the snapshots of our cosmological simulation at different redshifts, observing two aspects. First, we shift and rotate each snapshot to make it completely independent from the others. Second, we cut the boxes appropriately to avoid including matter from more than one snapshot, since contiguous snapshots partially overlap in redshift.

To create the maps, we proceed in two steps. We first project gas particles on a two-dimensional grid, and then stack the resulting planes summing the contribution from each individual plane to obtain the final map. Projecting the gas particles on a regular grid, we take the smoothing length of each particle into account and use the SPH (Smoothed Particle Hydrodynamics, see e.g. Monaghan 1992) interpolating kernel from the original simulation (Monaghan \& Lattanzio 1985). Particles are distributed over grid cells with a fractional weight calculated from the overlap of the spline kernel with the grid cell.

Since each particle has its individual smoothing length, the number of pixels involved in each projection varies, which produces appropriately smooth maps. This algorithm was applied by Puchwein et al. (2005) to study the impact of gas physics on strong lensing by clusters and by da Silva et al. (2000) to create simulated SZ maps.

We repeated this procedure for each snapshot of the simulation, and then summed the contributions of all planes to obtain the final map. We created eleven different maps including matter up to redshift $z=1$ and $z=2$ with a resolution of $2048 \times 2048$ pixels. The opening angle of the light cone is determined by the last plane of the pile, because its dimension is fixed in comoving units: it corresponds to 4.9 and 3.1 degrees, for light cones up to $z=1$ and $z=2$, respectively.

We built our light cones in the same way as for gravitationallensing maps, i.e. projecting the whole box on a regular grid and then selecting the portion of the plane enclosed by the light cone (see Fig. 1 in Pace et al. 2007). In other words, our planes all have the same number of pixels with constant comoving size, chosen according to the simulation resolution. Other authors (see e.g. da Silva et al. 2000; Roncarelli et al. 2006a) prefer to construct the light cones keeping the angular resolution of the pixels constant, such that each plane contributes the same amount of pixels. We avoid this because then the pixel resolution would exceed the physical resolution of the cosmological simulation, especially at low redshift.

\subsubsection{Simulating the SZ maps}

In order to create a map for the SZ effect we replace the lineof-sight integrals in Eqs. (1) and (4) by sums over gas particles. The contributions to the tSZ and kSZ effects of the $i$ th particle are thus

$y_{\mathrm{i}}=\frac{1}{L_{\mathrm{pix}}^{2}} \frac{k_{\mathrm{B}} \sigma_{\mathrm{T}}}{m_{\mathrm{e}} c^{2}} n_{\mathrm{e}, \mathrm{i}} T_{\mathrm{i}}$
$b_{\mathrm{i}}=\frac{1}{L_{\mathrm{pix}}^{2}} \frac{\sigma_{\mathrm{T}}}{c} n_{\mathrm{e}, \mathrm{i}} v_{\mathrm{r}, \mathrm{i}}$,

respectively, where $L_{\mathrm{pix}}$ is the physical size of a pixel at the distance of the corresponding plane. We approximate the radial velocity $v_{\mathrm{r}, \mathrm{i}}$ with the velocity component along the $z$-direction and relate the temperature of the gas particles $T$ (in Kelvin) to their internal energy per unit of mass $\left(U\right.$ in $\left.\mathrm{km}^{2} / \mathrm{s}^{2}\right)$ through

$T=10^{6} \times \frac{2}{3 k_{\mathrm{B}}} m_{\mathrm{p}} \mu U$

as for a monatomic ideal gas, where $m_{\mathrm{p}}$ is the proton mass and $\mu$ is the mean molecular weight

$\mu=\frac{1+4 y_{\mathrm{He}}}{1+y_{\mathrm{He}}+n_{\mathrm{e}}}$.

We adopt $y_{\mathrm{He}} \approx 0.08$.

\subsubsection{Simulating the $\mathrm{X}$-ray maps}

We create the light cone maps for the X-ray emission in the three energy bands mentioned above: soft $(0.5-2 \mathrm{keV})$, hard (2-4 keV) and hardest (4-10 keV). We model the contribution of each particle to the X-ray signal in the soft and hard bands adopting the MeKaL model (Mewe et al. 1995) as implemented in XSPEC (Arnaud 1996). It returns the emission spectrum of the hot diffuse plasma and is particularly suited for the soft band where the influence of the metal line emission is important. Using this model, the X-ray luminosity is

$L_{\mathrm{X}, \mathrm{i}}=\left(m_{\mathrm{p}} \mu\right)^{-2} m_{\mathrm{i}} \rho_{\mathrm{i}} x_{\mathrm{e}} \Lambda\left(T_{\mathrm{i}}, Z_{\mathrm{i}}, E_{1}^{\prime}, E_{2}^{\prime}\right)$,

where $x_{\mathrm{e}} \equiv n_{\mathrm{e}} / n_{\mathrm{H}}$ is the ratio between the number density of free electrons and hydrogen nuclei. The cooling function $\Lambda$ depends on the particle temperature, on the metallicity and on the energy band $\left[E_{1}, E_{2}\right]$.

For the hardest band, we can use the model described in Borgani et al. (1999) because the influence of metal lines is negligible there, and a simple power-law parametrisation suffices. It is then possible to parametrise the X-ray luminosity of the $i$ th particle in the hardest band by

$L_{\mathrm{X}, \mathrm{i}}^{\text {hardest }} \approx 1.7 \times 10^{42} \frac{m_{\mathrm{i}} \rho_{\mathrm{i}} \sqrt{T_{\mathrm{i}}}}{\mu^{2}} \mathrm{erg} / \mathrm{s}$, 


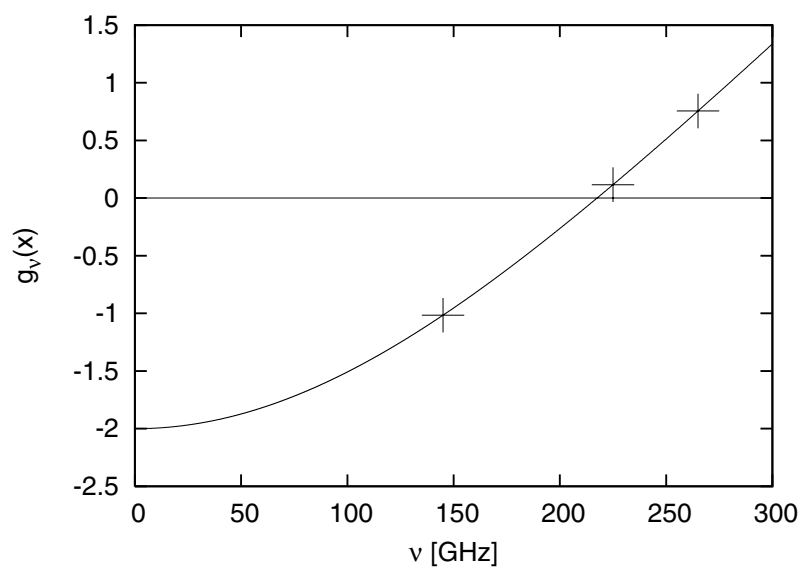

Fig. 2. The spectrum of the tSZ effect, with the ACT frequency bands overplotted (crosses).

where $m_{\mathrm{i}}, \rho_{\mathrm{i}}$ and $T_{\mathrm{i}}$ are its mass, its density, and its temperature in $\mathrm{keV}$.

For each gas particle, the X-ray flux is

$I_{\mathrm{X}, \mathrm{i}}=\frac{L_{\mathrm{X}, \mathrm{i}}}{4 \pi d_{\mathrm{L}}(z)^{2}} \mathrm{erg} / \mathrm{s} / \mathrm{cm}^{2}$,

where $d_{\mathrm{L}}(z)$ is the luminosity distance of the particle from the observer. As for the SZ maps, we project the X-ray flux on a regular grid using the SPH kernel.

\subsection{Simulating observations}

We simulate observations with specific instruments, namely the Atacama Cosmology Telescope (ACT) for the SZ effect because of its high angular resolution and sensitivity, and the space telescopes XMM-Newton and Chandra for the X-ray emission, assuming exposure times of 30 and $100 \mathrm{ks}$.

\subsubsection{Noise inclusion into the SZ map}

Figure 2 shows the tSZ spectrum $g_{v}(x)$. The crosses indicate the three frequency bands of ACT. The $v=225 \mathrm{GHz}$ channel is close to the frequency where $g_{v}(x)$ vanishes and the dominant signal will be due to the $\mathrm{kSZ}$ effect. Nonetheless, we shall see that this channel is very important for our multi-band frequency filter because it contains precious information on the noise due to the primary $\mathrm{CMB}$ anisotropies.

The noise in tSZ observations has three main contributions: the CMB radiation, the instrumental noise and the $\mathrm{kSZ}$ effect. The noise due to the primary $\mathrm{CMB}$ anisotropies and to the instrument can be modelled as two independent Gaussian random fields. We used the CMB power spectrum as computed with CMBEASY (Doran 2005), and the instrumental noise power spectrum

$C_{1}^{\text {noise }}=w^{-1} \exp \left[\frac{l(l+1) F W H M^{2}}{8 \ln 2}\right]$,

where $F W H M$ is the full width at half maximum of the instrumental beam in arcmins, $l$ represents the multipole order, and $w^{-1} \equiv(\Delta T / T F W H M)^{2}$. The quantity $\Delta T / T$ gives the sensitivity of the instrument on the scale of the beam (Knox 1995). Finally, the maps are convolved with the instrumental beam. Table 1 summaries some parameters used to mimic ACT observations.
Table 1. Parameters used to produce mock observations with ACT. In the first column we show the central bands frequencies at which ACT operates; second and third columns list the corresponding FWHM and sensitivity, respectively.

\begin{tabular}{ccc}
\hline $\begin{array}{c}\text { Central band frequency } \\
(\mathrm{GHz})\end{array}$ & $\begin{array}{c}F W H M \\
(\operatorname{arcmin})\end{array}$ & $\begin{array}{c}\Delta T / \text { beam } \\
(\mu \mathrm{K})\end{array}$ \\
\hline 145 & 1.7 & 2 \\
225 & 1.1 & 3.3 \\
265 & 0.93 & 4.7 \\
\hline
\end{tabular}

In Fig. 3 we show an example of the SZ simulations. The right panel displays the simulated observation with the $v=$ $145 \mathrm{GHz}$ channel of ACT, including the kSZ effect, the instrumental noise and the primary CMB fluctuations. The left and central panel show instead a simulated map for the tSZ and $\mathrm{kSZ}$ effect, respectively.

\subsubsection{Noise inclusion into the X-ray maps}

In order to transform our X-ray flux maps into count rates, we first multiply them by the energy conversion factor of the instrument. For each energy band, this factor is computed assuming a spectral model, consisting of thermal free-free emission with $k_{\mathrm{B}} T=4 \mathrm{keV}$, convolved with the response matrices of the EPIC-PN detector on-board XMM-Newton or with the ACIS-I array on-board Chandra. Assuming an exposure time (either 30 or $100 \mathrm{ks}$ ), we transform our count-rate maps into photon-count maps. We then added the background of an X-ray observation, given by the sum of the detector noise, the unresolved X-ray background, and the particle background. Finally, we convolved the resulting maps with the point-spread function (PSF) of the instrument (5 and 0.5 arcsec for XMM-Newton and Chandra, respectively). In order to reproduce the observational noise, we added Poissonian noise according to the local photon flux. The noise levels used in the simulated observations with XMM-Newton and Chandra are summarised in Table 2.

In Fig. 4 we give an example of X-ray simulation in the soft band. The left panel shows a synthetic X-ray map, while the corresponding simulated observation with XMM-Newton, including the background, instrumental and Poissonian noises is shown in the right panel. An exposure time of $30 \mathrm{ks}$ is adopted here. The maps are based on the same mass distribution underlying Fig. 3.

The difference in features between the X-ray and SZ maps reflect the different nature of these two physical processes. In contrast with the SZ effect where even the halos outskirts are visible, the clusters X-ray emission is much more concentrated because of the squared dependence on the gas density so that only the cluster core is visible. An example is given by the three bright objects in the upper right side of Fig. 4. They are low mass objects at low redshift, therefore they appear very diffuse in the SZ map and, only at first sight, prominent in the X-ray map, in fact the overall X-ray luminosity is indeed small. These differences complicate the association of a SZ detection to the corresponding X-ray one as will be discussed in Sect. 6.

\section{Filtering method}

We now describe the matched filter used to detect clusters in the simulated maps. For the X-ray observations, the noise is not correlated across bands or with the noise in SZ observations, thus the multi-band filter described above will be applied only to the SZ observations. 

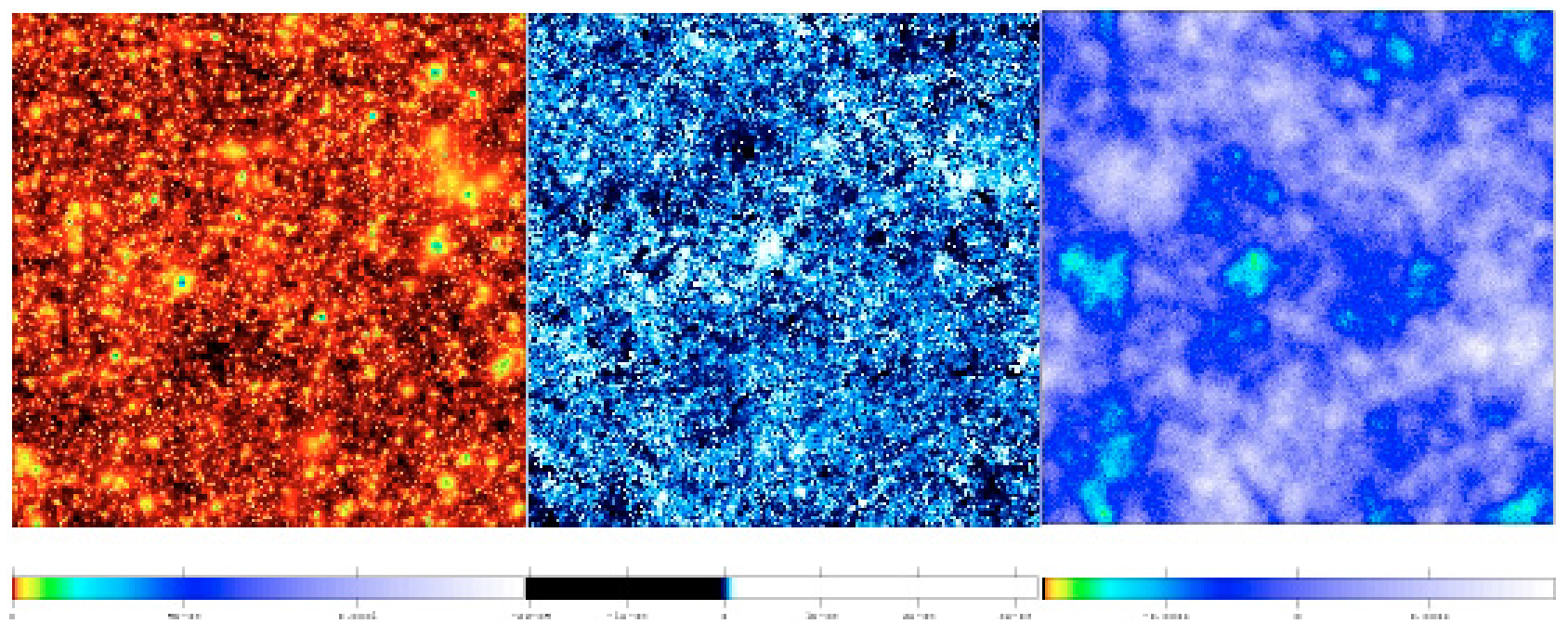

Fig. 3. Examples of SZ maps including cosmic structures to $z=1$. The left panel shows the simulated tSZ map, the central panel the kSZ map, and the right panel shows a simulated observation of the same field in the $145 \mathrm{GHz}$ channel of ACT, including the kSZ effect, the CMB signal and the instrumental noise. The size of the maps is $\approx 4.9 \mathrm{deg}$.

Table 2. XMM-Newton and Chandra noise levels and effective detector areas for the EPIC and ACIS-I instruments, respectively.

\begin{tabular}{cccc}
\hline \hline Instrument & Band & $\begin{array}{c}\text { Noise } \\
\text { (counts/s/deg }\end{array}$ & $\begin{array}{c}\text { Effective area } \\
\text { (at } 1.4 \mathrm{keV} \text { ) }\end{array}$ \\
\hline XMM-Newton & $0.5-2 \mathrm{keV}$ & 24.624 & \\
(EPIC-PN) & $2-4 \mathrm{keV}$ & 11. & $\sim 1200 \mathrm{~cm}^{2}$ \\
& $2-10 \mathrm{keV}$ & 19.64 & \\
\hline Chandra & $0.5-2 \mathrm{keV}$ & 2.96 & \\
(ACIS-I) & $2-4 \mathrm{keV}$ & 2.47 & $\sim 600 \mathrm{~cm}^{2}$ \\
& $4-10 \mathrm{keV}$ & 7.4 & \\
\hline
\end{tabular}

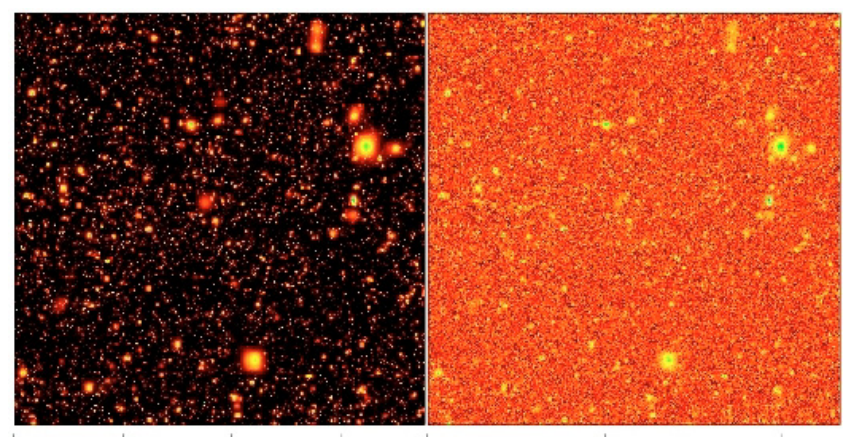

Fig. 4. Examples of X-ray maps. The left panel shows the X-ray flux in the soft band for the same field displayed in Fig. 3; the right panel represents a simulated observation with XMM-Newton assuming an exposure time of $30 \mathrm{ks}$. The size of the image is the same as in Fig. 3.

\subsection{Single-band filter}

The single-band filter $\Psi$ we use is analogous to the optimal filter proposed by Maturi et al. (2005). We suppose that the observational data $D(\boldsymbol{\theta})$ are given by

$D(\boldsymbol{\theta})=S(\boldsymbol{\theta})+N(\boldsymbol{\theta})$

where $S(\boldsymbol{\theta})=A \tau(\boldsymbol{\theta})$ is the signal from sources whose spatial shape is modeled by $\tau$, and $N(\boldsymbol{\theta})$ represents the noise. The estimated amplitude of the linearly filtered signal is

$A_{\mathrm{est}}(\boldsymbol{\theta})=\int \mathrm{d}^{2} \theta^{\prime} D\left(\boldsymbol{\theta}^{\prime}\right) \Psi\left(\left|\boldsymbol{\theta}^{\prime}-\boldsymbol{\theta}\right|\right)$.
Imposing the conditions that the optimal filter be unbiased $\left(\left\langle A_{\mathrm{est}}-A\right\rangle=0\right)$ and that its variance $\left(\sigma^{2}=\left\langle\left(A_{\mathrm{est}}-A\right)^{2}\right\rangle\right)$ be minimal, its shape follows from a variational problem with the two previously described constraints (see Haehnelt \& Tegmark 1996),

$\hat{\Psi}(\boldsymbol{k})=\frac{1}{(2 \pi)^{2}}\left[\int \frac{|\hat{\tau}(\boldsymbol{k})|^{2}}{P_{\mathrm{N}}(k)} \mathrm{d}^{2} k\right]^{-1} \frac{\hat{\tau}(\boldsymbol{k})}{P_{\mathrm{N}}(k)}$,

where the hats denote Fourier transforms. Equation (17) shows that the shape of the filter $\Psi$ is determined by the shape of the signal, $\tau$, and by the power spectrum of the noise, $P_{\mathrm{N}}$.

\subsection{A simple model for galaxy clusters}

We model the gas distribution for the filter template by the truncated King profile (King 1962)

$\rho(x)=\frac{1}{1+x^{2}} \sqrt{\frac{\left|x_{\mathrm{t}}^{2}-x^{2}\right|}{1+x^{2}}}$ for $x \leq x_{\mathrm{t}}$,

where $x=r / r_{\mathrm{c}}, x_{\mathrm{t}}=r_{\mathrm{t}} / r_{\mathrm{c}}, r_{\mathrm{c}}$ is the core radius, $r_{\mathrm{t}}$ is the truncation radius defined as ten times the virial radius $r_{\mathrm{v}}$. Outside $r_{\mathrm{t}}$ the gas distribution is zero. Although based on the simple assumption that gas follows a static and isothermal distribution, this profile is justified by simulations and X-ray observations (see e.g. Borgani et al. 2004; Finoguenov et al. 2001).

The tSZ effect (Eq. (1)) is proportional to the density $\rho$ and normalised to the Compton- $Y$ parameter

$Y=\int y \mathrm{~d} \Omega$

integrated over the solid angle $\Omega$.

The X-ray luminosity is proportional to the square of the electron density and thus to the square of the profile (18). It is normalised such that the bolometric luminosity follows the empirical relation

$L_{\mathrm{X}, \mathrm{bol}}=2.99 \times 10^{44} h^{-2}\left(\frac{T}{6 \mathrm{keV}}\right)^{2.6} \mathrm{erg} / \mathrm{s}$

(Kitayama \& Suto 1997), where $T=T_{\mathrm{vir}}\left(r_{\mathrm{s}}\right)$ is the X-ray temperature. The luminosity in a given band is computed using Eq. (6). We do not assume any explicit redshift dependence. 

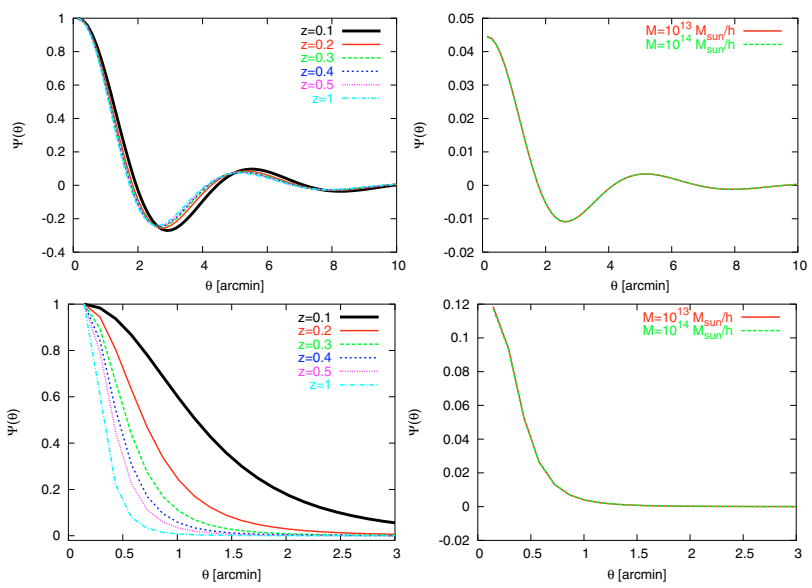

Fig. 5. The matched filter. The left panels show the filter profiles normalised to unity (for a better understanding) for different redshifts for a fixed halo mass of $M=10^{14} M_{\odot} / h$. The right panels show the filter profile for different halo masses at a fixed redshift of $z=0.5$. SZ and X-ray filter profiles are presented in the upper and lower panels, respectively.

\subsection{Filter dependence on the template}

Figure 5 shows the profile of the filter used for the SZ observations at $145 \mathrm{GHz}$ (upper panels) and for the X-ray observations in the soft band (lower panels). In the left panels, we assume a template with mass $M=10^{14} M_{\odot} / h$ and illustrate how the filter depends on the cluster redshift. In the right panels, we fix the redshift of the template to $z=0.5$ and show how the profile depends on cluster mass, based on the two cases $M=10^{13} M_{\odot} / h$ (solid line) and $M=10^{14} M_{\odot} / h$ (dashed line). The upper right panel demonstrates that the shape of the SZ filter is insensitive to both the mass and the redshift of the template, while only the normalisation changes.

This implies that its sensitivity is independent of the particular choice of the template. This characteristic results from the shape of the noise power spectrum, combined from instrumental noise and the CMB: since their power spectra are independent of both redshift and mass, the minimum of the noise (and therefore the maximum of the filter) is always at the same wavenumbers. Due to the particular shape of the noise power spectrum (shown in Fig. 6), the filter needs to oscillate because of the lack of information in a single band of the global characteristics of the background noise. Unlike the SZ filter, the X-ray filter (see the lower panels of Fig. 5) is simply proportional to the template because the noise is white. This implies that structures in the signal do not change. Thus, varying the template, the X-ray filter changes accordingly.

\subsection{The multi-band matched optimal filter}

The multi-band matched filter allows combining information from different bands. For its complete derivation, we refer to Schäfer et al. (2006) and Melin et al. (2006) and references therein. Generalising the single-band case, the data obtained at a given frequency $v$ are the sum of the signal $S_{v}(\boldsymbol{\theta})$ and the noise $N_{v}(\boldsymbol{\theta})$,

$D_{v}(\boldsymbol{\theta})=S_{v}(\boldsymbol{\theta})+N_{v}(\boldsymbol{\theta})$.

The signal is modelled as

$S_{v}(\boldsymbol{\theta})=A f_{v} \tau_{v}(\boldsymbol{\theta})$,

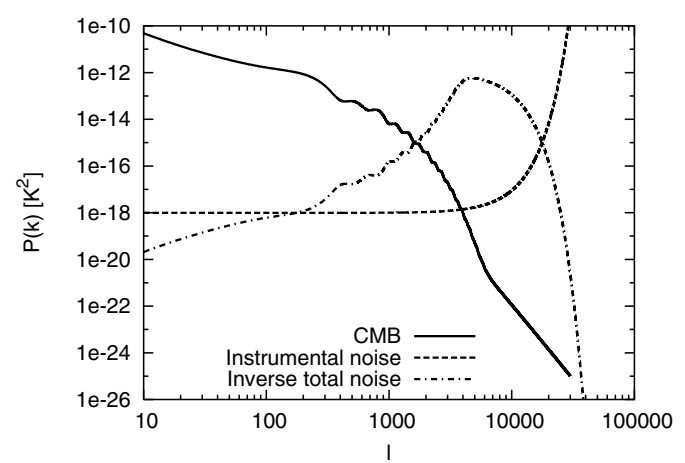

Fig. 6. The noise power spectra. The solid line shows the CMB power spectrum, the dashed line the instrumental noise, and the dotted-dashed line the inverse of the total noise, given by the sum of CMB and instrumental noise. The filter is proportional to the inverse of the total power spectrum.

where $A$ is the (band-independent) amplitude, $f_{v}$ is the frequency dependence of the amplitude and $\tau_{v}(\boldsymbol{\theta})$ is the spatial profile (normalised to unity). The index $v$ runs from 1 to the number of available bands, $M$. We assume that the background noise has zero mean in each band and that its statistical properties are fully characterised by the correlation functions

$C_{v_{1} v_{2}}=\left\langle\hat{N}_{v_{1}}(\boldsymbol{k}) \hat{N}_{v_{2}}\left(\boldsymbol{k}^{\prime}\right)^{*}\right\rangle=(2 \pi)^{2} \delta\left(\boldsymbol{k}-\boldsymbol{k}^{\prime}\right) P_{1, v_{1} v_{2}}(k)$,

where $P_{1, v_{1} v_{2}}(k)$ is the cross-power spectrum.

To measure the signal amplitude $A$, we define a linear estimator for each band,

$A_{\mathrm{est}, v}(\boldsymbol{\theta})=\int \mathrm{d}^{2} \theta^{\prime} D_{v}\left(\boldsymbol{\theta}^{\prime}\right) \Psi_{v}\left(\left|\boldsymbol{\theta}^{\prime}-\boldsymbol{\theta}\right|\right)$

and a total estimate by

$A_{\mathrm{est}}=\sum_{\nu=1}^{M} A_{\mathrm{est}, \nu}(\boldsymbol{\theta})$.

In analogy with the single-band filter, $\boldsymbol{\Psi}(\boldsymbol{\theta})=\left[\boldsymbol{\Psi}_{\boldsymbol{v}}(\boldsymbol{\theta})\right]$ is the optimal filter, minimising the estimated variance, $\sigma^{2}=\left\langle\left(A_{\text {est }}-\right.\right.$ $\left.\langle A\rangle)^{2}\right\rangle$, and avoiding bias, $b \equiv\left\langle A_{\mathrm{est}}-A\right\rangle=0$.

In deriving the filter, it is convenient to define the filter vector $\bar{\Psi}(\boldsymbol{\theta})=\left[\Psi_{v}(\boldsymbol{\theta})\right]$ and the signal vector $\overline{\boldsymbol{F}}(\boldsymbol{\theta})=\left[F_{v}(\boldsymbol{\theta})\right]$. We further define the cross-power matrix $\boldsymbol{C}^{(l)}=\left[C_{v_{1} v_{2}}^{(l)}\right]$.

Schäfer et al. (2006) show that the filter satisfying these conditions is

$\Psi=\alpha \boldsymbol{C}^{-1} \boldsymbol{F}$,

where $\boldsymbol{C}^{-1}$ is the inverse of the matrix $\boldsymbol{C}$, and the normalisation factor $\alpha$ is given by

$\alpha^{-1}=\int \mathrm{d}^{2} k \mathbf{F}^{\mathrm{T}} \mathbf{C}^{-1} \mathbf{F}$

The estimate has the variance

$\sigma^{2}=\int \frac{\mathrm{d}^{2} k}{(2 \pi)^{2}} \Psi^{\mathrm{T}} \boldsymbol{C} \Psi$.

According to Eq. (26), the matched filter depends on the noise, taking advantage of its correlation between all bands. In the special case $C_{v_{1} v_{2}}=C_{v_{1}} \delta\left(v_{1}-v_{2}\right)$, i.e. in the case of uncorrelated noise, the matrix $\boldsymbol{C}$ and thus also its inverse are diagonal, and 


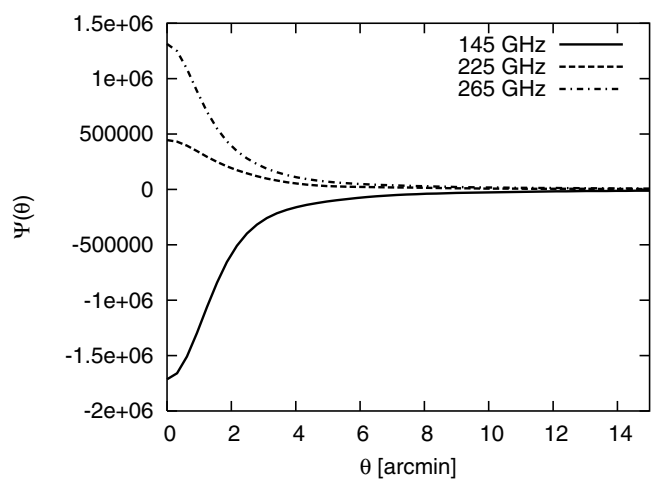

Fig. 7. The profile for the matched tSZ filter for a halo template with $M=10^{14} M_{\odot} / h$ at $z=0.4$. The solid, dashed and dash-dotted lines refer to the $145 \mathrm{GHz}, 225 \mathrm{GHz}$ and $265 \mathrm{GHz}$ bands, respectively.

the optimal filter for each band had the same shape as the singleband filter defined in Sect. 4.1. Just the normalisation differs.

Figure 7 shows the matched-filter profile for the three bands of ACT and a template with $M=10^{14} M_{\odot} / h$ at $z=0.4$. It is evidently broader than for a single band (see Fig. 5). Note also that the multi-band SZ filter does not oscillate because the multi-band filter has complete information on how to separate the signal from the noise pattern and therefore does not need to compensate the background by oscillations. The matched filter described in Schäfer et al. (2006) shows oscillations (see Fig. 6) because a different noise power spectrum was used to define the filter, in particular Galactic foregrounds were added.

\section{Results: statistics of the detections}

We now apply the single- and multi-band filters to our set of eleven SZ and X-ray simulated maps and statistically analyse the samples obtained. We define a detection as a group of neighbouring pixels in the signal-to-noise (hereafter $\mathrm{S} / \mathrm{N}$ ) maps, whose values exceed a certain threshold. The pixel with the largest $S / N$ value defines its position. A detection is true if it can be associated to a halo present in the original $\Lambda \mathrm{CDM}$ simulation, while it is spurious if caused by noise.

In order to reliably associate each detection with a halo from the parent simulation, we apply the method described and used in Pace et al. (2007), to which we refer for details. Briefly, we produce sequences of maps by removing sequentially one plane from the stack. If a halo is in fact located on an individual plane, its detection must disappear from the map when this plane is removed. If a detectable peak keeps appearing in all maps of a sequence, it must originate from the noise.

\subsection{SZ single-band detections}

Due to the oscillations of the single-band filter (see the upper panels of Fig. 5), detections consist of positive $S / N$ peaks surrounded by negative and positive ring-like structures. Typically, these rings are fragmented by noise and appear as secondary $S / N$ peaks surrounding the most prominent detections. These secondary peaks can easily be confused with true detections. Some examples can be seen in the left panel of Fig. 8. Consequently, we need to include these correlated noise structures in our noise model.

This is possible because we can easily estimate their expected shapes and amplitudes, which derive from the convolution of the signal with the oscillatory pattern of the filter (see
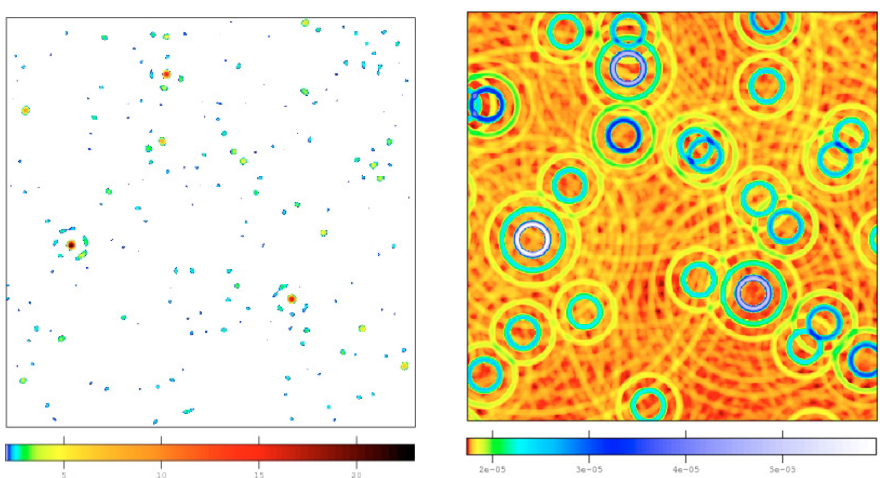

Fig. 8. Maps for the $S / N$ ratio illustrating the procedure to suppress artificial structures in the filtered SZ maps using the correlated noise. The left panel shows the detections with $S / N \geq 2$ as obtained with the filter, while the right panel shows the noise map including the damped oscillations of the single-band filter.

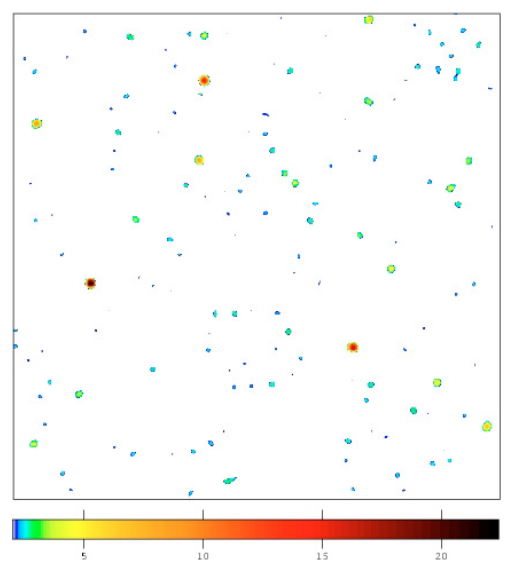

Fig. 9. $S / N$ map, as shown in Fig. 8, after removal of the spurious ring artifacts. The side length of the map is 2.5 degrees.

Fig. 5). The noise map is thus obtained adopting an iterative approach. First, we compute the $S / N$ maps by applying the filter and estimating the noise. Second, we identify the detection having the highest $S / N$ value, compute the correlated noise pattern as explained above and include it into the noise map. Then, we use this new noise estimate to compute an updated $S / N$ map, where we identify new detections searching for local $S / N$ maxima. We can iterate this procedure until no more detection is found with $S / N$ exceeding a given threshold. The noise map resulting from this procedure is shown in the right panel of Fig. 8. The resulting $S / N$ map obtained using the correlated noise pattern is shown in Fig. 9, where, thank to the use of the correlated noise, we notice that the fragmented rings are efficiently suppressed.

The upper panels of Fig. 10 show the total number of detections per square degree in our ACT simulations. Results are shown for all three channels and for light cones limited to $z=1$ and $z=2$ (left and right panels, respectively). The corresponding fractions of spurious detections are displayed in the bottom panels. Results are averaged over eleven maps covering $24.5 \mathrm{deg}^{2}$ and $9.5 \mathrm{deg}^{2}$ and renormalized to $1 \mathrm{deg}^{2}$ for limiting redshifts $z=1$ and $z=2$, respectively. As expected, we find very few detections at $v=225 \mathrm{GHz}$ where the tSZ effect is very weak.

Since the SZ effects depend only mildly on redshift, we find more objects in a light cone extending up to redshift $z=2$ than for $z=1$. In particular, at $v=145 \mathrm{GHz}$ and $v=265 \mathrm{GHz}$, 

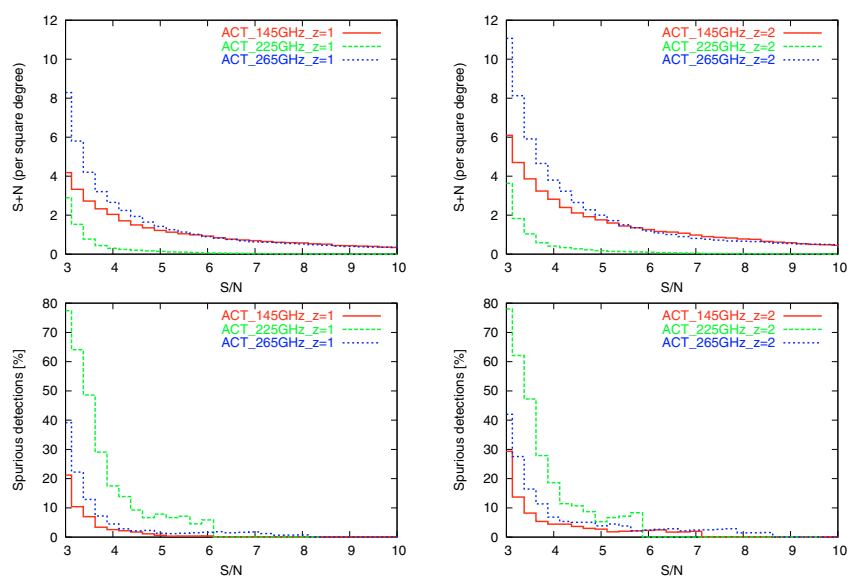

Fig. 10. The total number of detections per square degree (upper panels) and the fraction of spurious detections (bottom panels) as functions of the $S / N$ ratio. The results are averaged over eleven realisations and shown for light cones extending to redshifts $z=1$ (left panels) and $z=2$ (right panels). Different line styles refer to the different bands considered, as indicated in the plots.

we find at least $30 \%$ more detections in the deeper light cone. The main differences for the results in these two bands are at low signal-to-noise ratios, where more structures are detected in the highest-frequency band, owed to the higher sensitivity of the instrument. For $S / N \geq 5$, the number of detections is similar in the two bands.

Because of the low tSZ signal, the fraction of spurious detections at $v=225 \mathrm{GHz}$ is very high $(\approx 80 \%$ for $S / N \approx 3)$ and virtually independent of the limiting redshift of the light cones. For the maps at $v=145 \mathrm{GHz}$ and $v=265 \mathrm{GHz}$ at $z=1(z=2)$, we find that $20 \%$ and $40 \%$ (30\% and $40 \%)$ of the detections at $S / N \approx 3$ are spurious, respectively. For $S / N>4$, the fraction of spurious detections drops to a few per cent. Including matter up to redshift $z=2$ into the light cones slightly increases the fraction of spurious detections because unresolved structures overlap. The number of detections with high $S / N$ does not increase with the depth of the light cones because the massive structures responsible for them are mainly located at low redshifts, but the number of low- $S / N$ detections is increased by up to a factor of five for the least massive detectable halos.

The upper panels of Fig. 11 show the fraction of halos detected in different bands as a function of mass. The left and right panels show the results for light cones up to $z=1$ and $z=2$, respectively. The values shown are obtained after averaging over eleven realisations. The lower panels of Fig. 11 present the sensitivity of the detections, expressed in terms of the minimum detectable mass as a function of redshift. To reduce the noise, this was computed combining two subsequent planes in the light cone and averaging the mass of the ten least massive halos detected in the corresponding redshift interval.

Since the tSZ effect depends only mildly on the source redshift, the completeness for detections up to $z=1$ and $z=2$ is essentially the same. The two channels at $145 \mathrm{GHz}$ and $265 \mathrm{GHz}$ perform equally well, while the completeness is much lower at $225 \mathrm{GHz}$, as expected. The minimum detected mass is $M \simeq 7 \times 10^{13} M_{\odot} / h$ and the completeness reaches $100 \%$ for masses $M \simeq 3 \times 10^{14} M_{\odot} / h$. The curve is quite steep, showing that the completeness drops rapidly as the mass decreases. For halo masses $\geq M \simeq 1.5 \times 10^{14} M_{\odot} / h$, the completeness has already dropped to $\sim 50 \%$. The minimum detectable halo mass is essentially constant for all $z$, reflecting the near-independence of
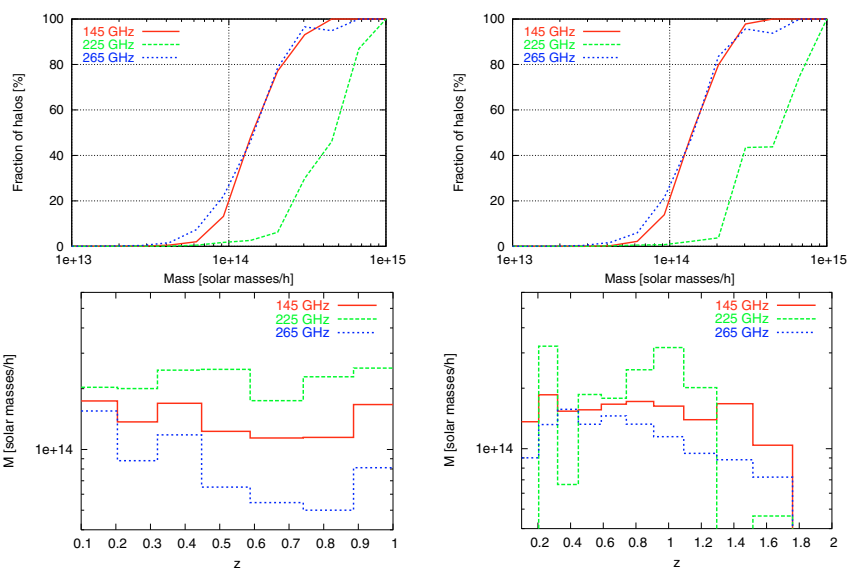

Fig. 11. Upper panels: the fraction of detected halos as a function of the halo mass. Lower panels: the sensitivity of the method expressed in terms of the minimum detectable mass as a function of redshift. Results are shown for light cones extending to $z=1$ and $z=2$ in the left and right panels, respectively. Different line styles refer to the three different ACT bands, as labeled. The curves represent average over eleven realisations.

the tSZ effect on distance. Again, the method is much less sensitive at $225 \mathrm{GHz}$ than at the two other frequencies. In addition, the filter tends to find halos with lower mass at $265 \mathrm{GHz}$ than at $145 \mathrm{GHz}$ (blue and red curves, respectively), explaining the higher completeness in the former band. This is due to the fact that the band at $265 \mathrm{GHz}$ has a better FWHM (see Table 1) and the noise is therefore lower (see Eq. (14)).

Similar results were found by López-Caniego et al. (2006) using the same approach to detect clusters in simulated Planck data. For low detection limits (corresponding to low $S / N$ ratios), López-Caniego et al. (2006) found a very high fraction of spurious detections, decreasing quite rapidly towards higher flux limits. However, results can only be compared at a qualitative level because the instrument and the band frequencies differ greatly. In particular, only two channels used by López-Caniego et al. (2006) have a frequency range close to those of ACT. Moreover, we study $S / N$ ratios rather than fluxes and account for the correlated ring patterns due to the oscillatory filter behaviour in the noise model.

\subsection{Statistics of $S Z$ multi-band detections}

Figure 12 shows an example of the $S / N$ maps obtained by applying each component of the multi-band filter to the three channels at $145 \mathrm{GHz}$ (upper left panel), $225 \mathrm{GHz}$ (upper right panel) and $265 \mathrm{GHz}$ (bottom left panel). The final result is obtained by combining them using Eq. (25) to give the $S / N$ map of the multi-band detection in the bottom right panel.

Compared to the results obtained with the single-band filter, the $S / N$ ratios are now enhanced by at least a factor of three. As before, the $S / N$ peaks in the maps were identified with SExtractor (Bertin \& Arnouts 1996) which allows easy deblending of nearby detections. The number counts per square degree as a function of the $S / N$ threshold are shown in the upper left panel of Fig. 13. The solid and dashed lines refer to light cones extending up to $z=1$ and $z=2$, respectively.

The lower number of total detections respect to the singleband filter at $265 \mathrm{GHz}$, has not to be interpreted as a signal of a poorer performance of the multi-band filter, but it has to be ascribed partly to the fact that the multi-band filter is wider 

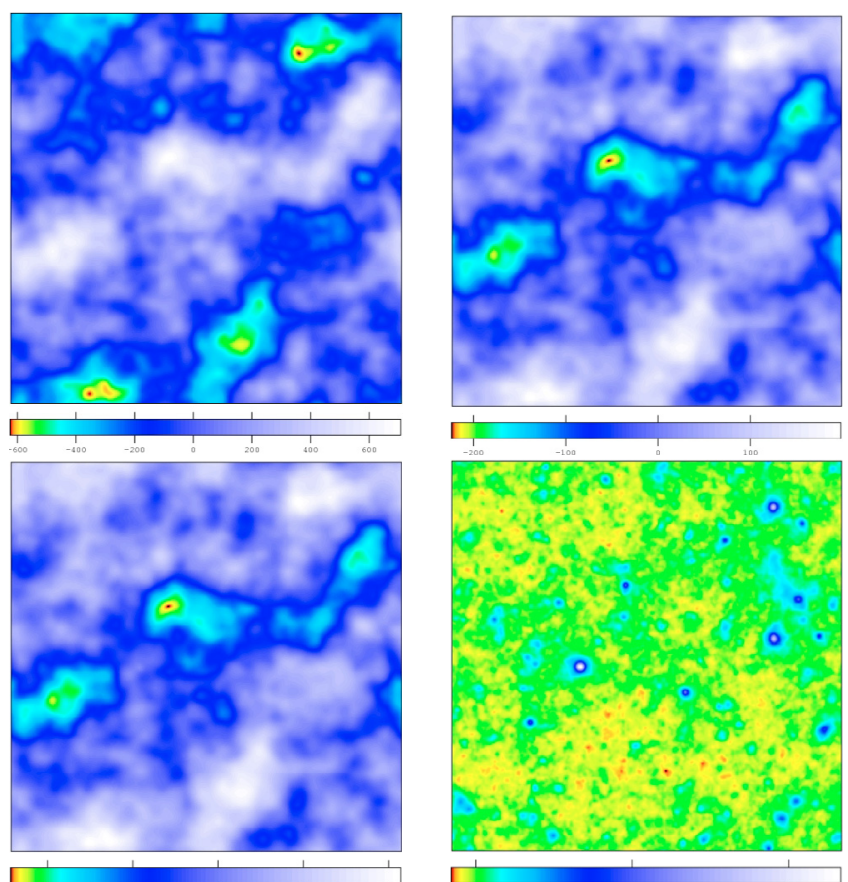

Fig. 12. Example of the application of the multi-band matched filter to a $S / N$ map. The starting input is the noisy map in the right panel of Fig. 3. Different panels refer to the maps filtered at different frequencies: $145 \mathrm{GHz}$ (upper left panel), $225 \mathrm{GHz}$ (upper right panel), $265 \mathrm{GHz}$ (lower left panel). The final $S / N$ map obtained by summing the previous three maps is shown in the lower right panel.
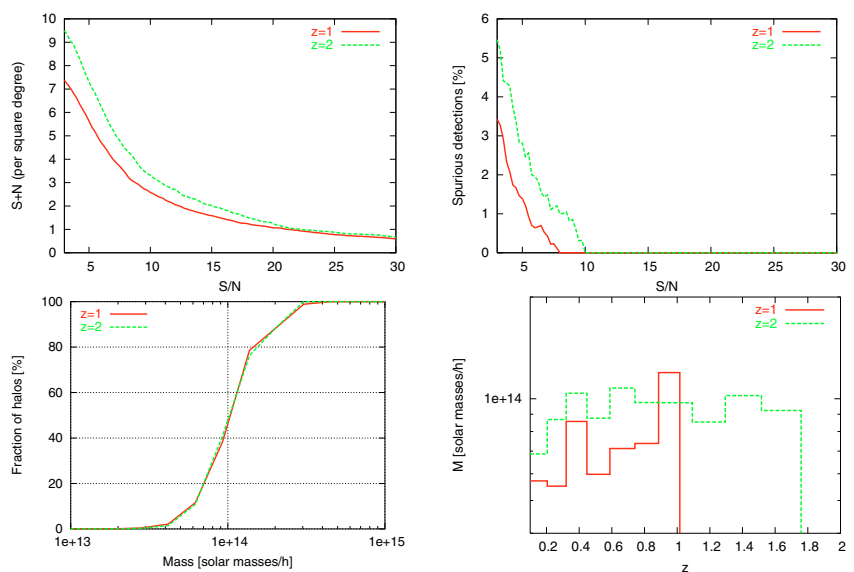

Fig. 13. Upper left panel: the total number of detections per square degree as a function of the $S / N$ ratio, obtained with the multi-band filter. Upper right panel: the fraction of spurious detections as a function of the $S / N$ ratio. Bottom left panel: the fraction of halos detected as a function of mass. Bottom-right panel: the minimum detectable mass as a function of redshift. Solid and dotted curves show results for light cones extending to $z=1$ and $z=2$, respectively.

and increases the confusion which is not entirely removed by SExtractor, but most importantly because of the lower sample contamination. In fact, the fraction of spurious detections is much lower for the multi-band than for the single-band filter. We find that the number of real, multi-band detections is higher by almost one order of magnitude for $S / N \approx 3$ and by a factor of five for $S / N \approx 5$ than the single-band detections. These results do not depend on the depth of the light cones.

The top-right panel of Fig. 13 shows the fraction of spurious detections in the final catalogue. Again, the great advantage of multi-band observations is obvious. While the fraction of spurious detections in the $265 \mathrm{GHz}$ channel alone is $\sim 40 \%$ at $S / N=3$ and $\sim 5 \%$ at $S / N \sim 5$, multi-band filtering reduces the fraction of spurious detection to the level of a few percent even at very low $S / N$ ratios. Thus, the vast majority of the detections in the top-right panel is due to real structures. The completeness of the catalogue is shown in the bottom left panel of Fig. 13. We find that $20 \%, 50 \%$ and $80 \%$ of the halos with masses exceeding $7 \times 10^{13} M_{\odot} / h, \sim 10^{14} M_{\odot} / h$ and $2 \times 10^{14} M_{\odot} / h$ contained in the light cones are detected by the multi-band filter, respectively. In contrast, the single-band filter detects only $\sim 20 \%$ of the halos with masses exceeding $10^{14} M_{\odot} / h$ in the $145 \mathrm{GHz}$ and in the $265 \mathrm{GHz}$ channels. Finally, we present in the bottom right panel of Fig. 13 the minimum halo mass detectable for the multiband filter as a function of redshift. Again, the curves show a very weak dependence on redshift, although the values seem to weakly increase with redshift. The mass threshold ranges from $\sim 6 \times 10^{13} M_{\odot} / h$ at $z=0$ to $\sim 9 \times 10^{13} M_{\odot} / h$ at $z=1.8$, which confirms that the multi-band filter also improves the sensitivity.

We conclude this section by comparing our results to an independent study of multi-band filtering. Melin et al. (2006) adopted a multi-band matched filter for analysing Monte-Carlo simulations. Mimicking SZ observations with the South-PoleTelescope (SPT), they performed a statistical study of the detections comparable to ours. Since the SPT bands and FHWM are similar to those of ACT, a fair comparison between our and their results is possible. They found 6 detections per square degree at $S / N>5$, very close to the 8 detections per square degree we find. The lowest masses they can detect is three times higher than we find for ACT. This is expected because of the higher noise level of SPT compared to ACT. Our spurious detection rate is similar to what found by Melin et al. (2006) and Sehgal et al. (2007). Only the latter has a slightly lower completeness $\approx 90 \%$ for masses $M \geq 3 \times 10^{14} M_{\odot} / h$ because they accounted the contamination of point sources and dust emission, and because they used a multi-frequency Wiener filter.

\subsection{Statistics of $X$-ray detections}

We now turn to the halo detections in the simulated X-ray maps. We show results only for light cones extending up to $z=1$, because the number of X-ray sources at higher redshifts is very low, and the corresponding plots are very similar to those for $z=1$.

The left panels of Fig. 14 show the total number of detections per square degree. From top to bottom, results are shown for the soft, hard and hardest bands. They represent the average over eleven different map realisations and numbers are normalised to $1 \mathrm{deg}^{2}$. The number of detections found for XMM-Newton is at least five times higher than with Chandra. Although XMM-Newton has a higher background level than Chandra, its better performance is due to the different effective area of the instrument (see Table 2). The number of detections decreases quite rapidly with the $S / N$ threshold. We find few objects with high signal-to-noise ratios also in the high-energy band.

The fraction of spurious detections is presented in the right panels of Fig. 14. We notice that it is always below $20 \%$ in all bands for detections with $S / N \gtrsim 3$, and that it drops to a constant value $\approx 5 \%$ independent of exposure time and instrument. This is due to few spurious peaks having quite high $S / N$ ratio. Since also the number of real objects with high $S / N$ ratio is approximately constant over a large range of $S / N$, their ratio is almost constant. These few, but prominent spurious detections 

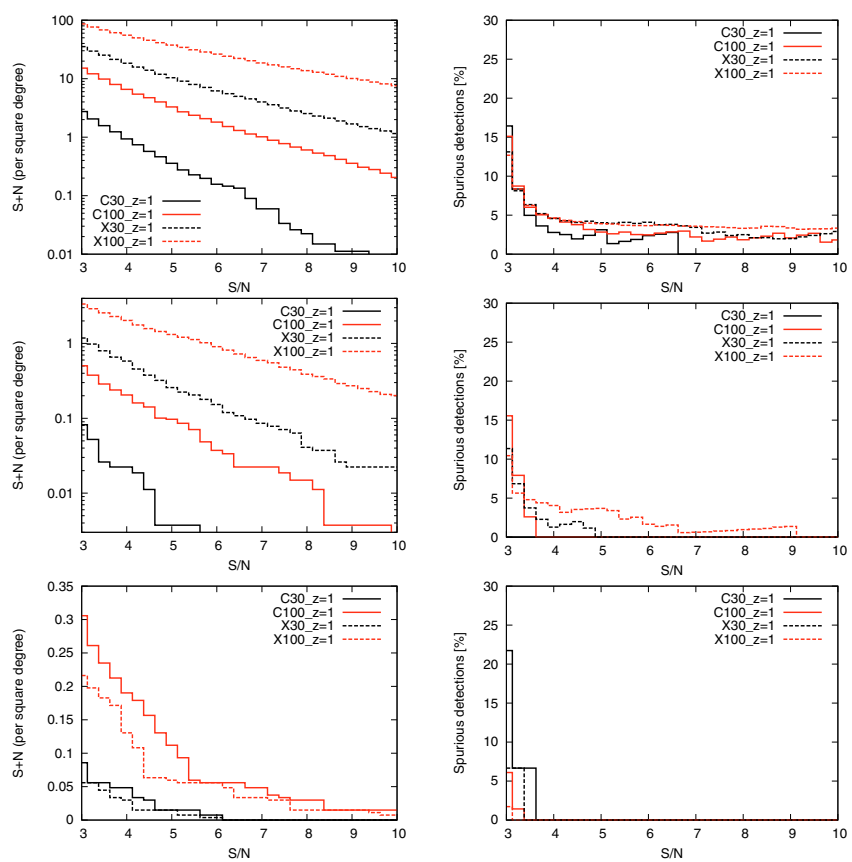

Fig. 14. Total number of X-ray detections per square degree (left panels) and fraction of spurious detections (right panels) as a function of the $S / N$ ratio. The results are shown for three different bands analysed: soft band (upper panel), hard band (middle panel), hardest band (bottom panel). The light cones extend to $z=1$. Different line styles correspond to Chandra and XMM-Newton, respectively), different colours to different exposure times (black and red curves are for $30 \mathrm{ks}$ and $100 \mathrm{ks}$, respectively). The results are averaged over eleven realisations.

are due to regions in the maps where several low-mass halos are approximately aligned along the line-of-sight.

Figure 15 shows the completeness of the detections. In the soft and hard bands, the detection catalogues for XMM-Newton are always more complete than for Chandra. In the soft band, the completeness decreases much more slowly as a function of mass than in the harder bands. The completeness reaches $100 \%$ for masses $M \gtrsim 2 \times 10^{14} M_{\odot} / h$, and it is $\sim 50 \%$ at $M \sim 3 \times 10^{13} M_{\odot} / h$ for XMM-Newton with an exposure time of $100 \mathrm{ks}$, while it is of the order of few percent for Chandra. In the hard band, Chandra detects only halos with mass $M>2-3 \times 10^{14} M_{\odot} / h$, and the detection threshold drops by a factor four with XMM-Newton. Results for the soft band are similar, but the softer photons of lower-mass halos lower the detection limit to $M \approx 10^{13} M_{\odot} / h$. Conversely, only halos with masses exceeding a few times $10^{14} M_{\odot} / h$ can be detected in the hardest band. There, the completeness for Chandra is almost as high or even larger than for XMM-Newton for an integration time of $100 \mathrm{ks}$, because only very massive halos are detected, and Chandra has a better resolution than XMM-Newton (see Table 2). As the X-ray emission depends mostly on the particles of the halo core, it might be that X-ray properties for halos of mass $M \approx 10^{13} M_{\odot} / h$ are not very well converged, therefore reliable results can only be safely inferred to halos of mass $M \approx 5 \times 10^{13} M_{\odot} / h$. The limit is shown in the left panels of Fig. 15 by the black vertical solid line.

The right panels of Fig. 15 show the sensitivity for the $\mathrm{X}$-ray detections. In the soft band, Chandra performs worse than $\mathrm{XMM}-$ Newton and thus requires larger masses for detection. In the hard and hardest bands, the sensitivity at low redshifts is similar for both satellites, even if Chandra performs slightly better because of its better resolution. Chandra finds halos only up to
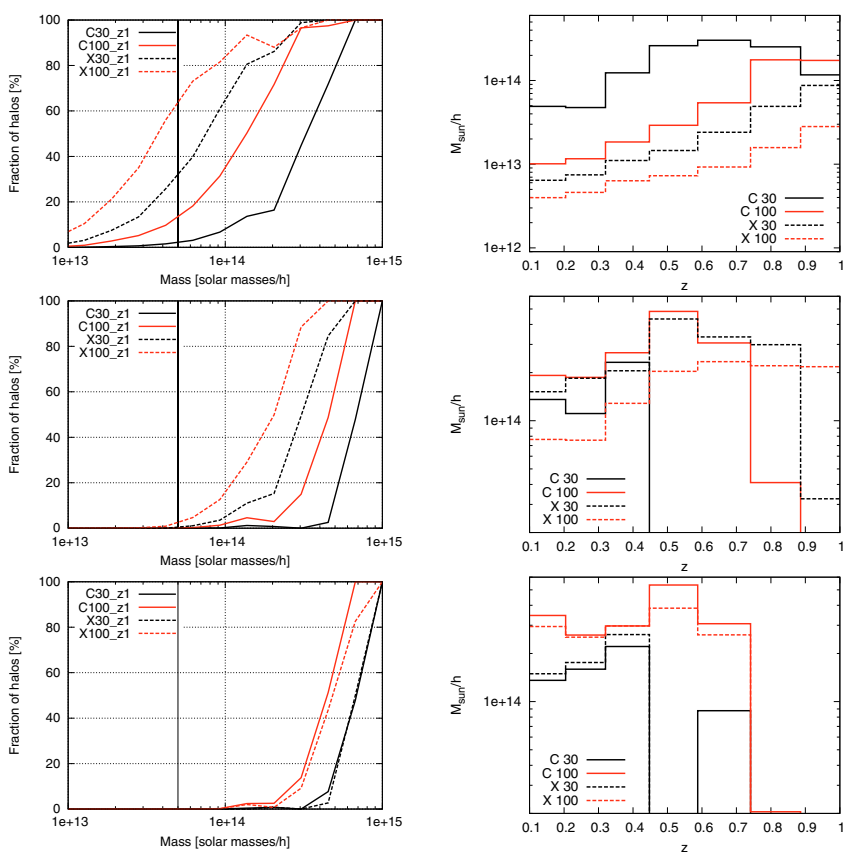

Fig. 15. Fraction of halos detected as a function of halo mass (left pan$e l s$ ) and sensitivity of the method (expressed in terms of minimum detectable mass, right panels) in three different X-ray bands: soft band (upper panel), hard (middle panel), and hardest (bottom panel). Light cones extend to $z=1$. Line-styles and colours refer to different instruments and exposure times, as in Fig. 14. The results are averaged over eleven realisations.

$z \sim 0.4$ in the hard band (for an integration time of $30 \mathrm{ks}$ ), while XMM-Newton reaches $z \sim 0.9$.

As expected, only the most massive clusters can be identified at high redshifts. In particular we note that the detection threshold increases approximately by a factor of 5 between $z \approx 0.1$ and $z \approx 1$. The mass of detected halos also increases in the hard and hardest bands because only the most massive halos emit such energetic photons. Finally, we recall that multi-band filtering is pointless for X-ray analyses because the noise in the different bands is uncorrelated, hence a multi-band filter would reduce to a single-band filter.

Compared to Finoguenov et al. (2007), who used a wavelet scale-wise reconstruction of the image (for details see Vikhlinin et al. 1998), we find a higher contamination in our sample, due to the fact that unlike Finoguenov et al. (2007) we do not use any optical information, but the amount of X-ray detections with $S / N>3$ is similar.

\section{Correlation between X-ray and multi-band SZ detections}

We now describe the properties of common detections in the $\mathrm{X}$-ray and SZ maps. We only consider X-ray detections in the soft band because their number was highest, and correlate them with multi-band SZ detections. A major problem is caused by the different angular scales of the detections in the different maps. Objects detected in X-rays are much smaller and more numerous. Thus, many of them may overlap with the same SZ detection according to the exposure time. For XMM-Newton with an integration time of $100 \mathrm{ks}$, we found that a single SZ detection on average overlaps with at least ten X-ray detections. To solve this problem we only considered the brightest X-ray detection contained in the portion of the map associated to the SZ detection. 

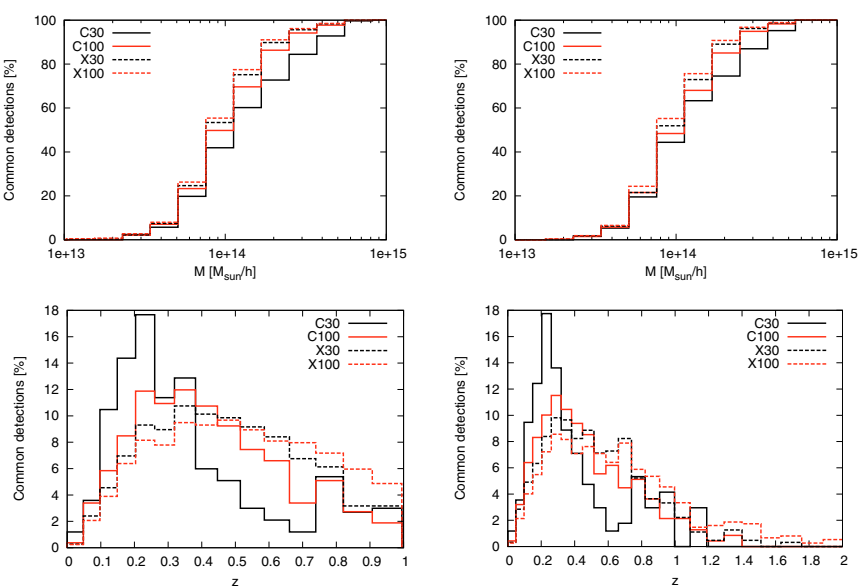

Fig. 16. Top panels: cumulative mass distribution for halos detected in both X-rays and SZ maps. Results are shown for light cones extending to $z=1$ (left panels) and $z=2$ (right panels). Bottom panels: redshift distributions for the same halos. The solid line refers to Chandra, the dotted line to XMM-Newton. The black and red curves refer to the two different integration times (30 and $100 \mathrm{ks}$, respectively).

Figure 16 shows the percentage of true detections in the $\mathrm{X}$-ray observations which have a SZ counterpart. We show the cumulative percentage as a function of halo mass (top panels), and a differential distribution as a function of redshift of the detected halos (bottom panels). The left and right panels refer to light cones with limiting redshifts $z=1$ and $z=2$, respectively. Different line styles correspond to different instruments in the X-ray observations, while different colors refer to different integration times. The curves are normalised to the total number of clusters present in both X-ray and SZ catalogues. The mass distribution of the common detections reflects the mass sensitivity of the SZ multi-band filter (see Fig. 13), while the redshift selection reflects the dependence of the X-ray sensitivity on redshift, which drops towards high redshift.

In the lower panels of Fig. 16, we notice that the maximum fraction of common detections occurs approximately at $z=0.3$, due to the X-ray sensitivity. Several X-ray detections will be masked by a single SZ detection and thus not seen. This implies that combining SZ with X-ray maps will result in a halo number strongly biased by the angular extent of the SZ detection. A given SZ halo will mask X-ray clusters projected into its close neighbourhood, independently of their redshift. This can be problematic for studies of substructures because it is impossible to identify substructures in the SZ map, and it will become difficult to study clusters at intermediate redshifts. This will be addressed in more detail in future work.

The upper panels of Fig. 17 show the correlation between the $\mathrm{X}$-ray and tSZ signals of the common real detections grouped according to their $S / N$ ratio significance, overlaid with the theoretical SZ and X-rays correlation as obtained in the original simulation. The analysis is limited to the soft band for XMM-Newton and an exposure time of $100 \mathrm{ks}$.

As expected, detections with $S / N>3$ occupy the upper right part of the contours $\left(y>10^{-6}\right.$ and $\left.X>10^{-11} \mathrm{erg} / \mathrm{s} / \mathrm{cm}^{2} / \mathrm{deg}^{2}\right)$ because they correspond to the most massive (and therefore brighter) halos. We also note that we do not find any detection with $y<10^{-7}$ and $X<10^{-13} \mathrm{erg} / \mathrm{s} / \mathrm{cm}^{2} / \mathrm{deg}^{2}$ because these values of the flux correspond to objects whose mass is of the order of the minimum mass that the filters can detect.

The lower panels show, the correlation between the filtered maps (y-axis) and the original maps ( $x$-axis). On the left, we
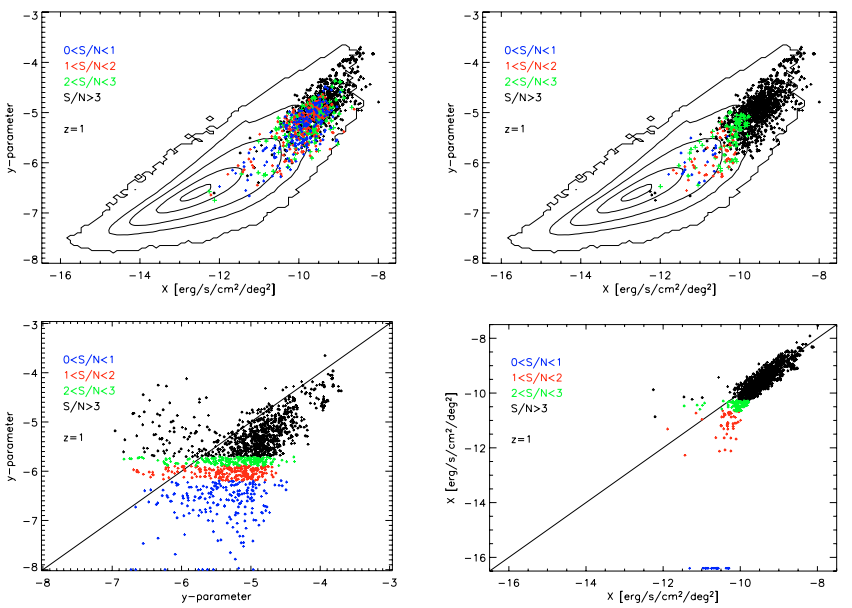

Fig. 17. Upper panels: correlation between the tSZ and X-ray signals ( $y$ and $x$-axes, respectively). The results are compared to the distribution of common detections, and are shown as a function of their $S / N$ ratios (blue for $0<S / N<1$, red for $1<S / N<2$, green for $2<S / N<3$, and black for $S / N>3$ ). The left and right panels refer to the $S / N$ values obtained using the multi-band SZ and the (soft-band) X-ray filter, respectively. Lower panels: correlation between the values in the filtered maps ( $y$-axis) and the original ones ( $x$-axis) for the multi-band SZ filter (left panel) and the (soft-band) X-ray filter (right panel). Again detections are binned using their $S / N$ ratios. The diagonal line would correspond to a perfect agreement between original and filtered maps. All plots refer to results for light cones extending up to $z=1$.

show the SZ multi-frequency filter, on the right the X-ray maps. We notice a substantial scatter for low Compton- $y$ parameters in the SZ compared to the X-ray maps, corresponding to detections with very low $S / N$ ratio. We also note that, while the filtered $\mathrm{X}$-ray maps show unbiased values for $S / N>3$, this is not strictly true for the multi-band SZ filter: the estimated amplitudes are biased towards lower values. This may be due to the fact that the $\beta$-model does not exactly reproduce the signal distribution of the SZ-halos. Finally, we found that the signal-to-noise ratios of the common SZ and X-rays detections are uncorrelated.

\section{Conclusions}

We have tested the performance of linear filtering in optimising and combining galaxy-cluster detections in X-ray and SZ observations. Based on a large $N$-body hydrodynamical cosmological simulation, we have studied the halo selection function, the contamination of halo catalogues and the mass sensitivity of the filtering technique. Our work extends earlier studies by taking gas physics properly into account and treating SZ and X-ray objects simultaneously with similar techniques.

We constructed light cones up to redshifts 1 and 2 and simulated typical observations of the X-ray emission (modelled after the XMM-Newton and Chandra observatories) and the SZ signal (adapted to ACT) produced by the matter contained in the light cones. Our set of 11 different realisations covers total areas of $\sim 264 \mathrm{deg}^{2}$ and $\sim 106 \mathrm{deg}^{2}$ for limiting redshifts $z=1$ and $z=2$, respectively.

We constructed and used single- and multi-band matched filters for halo detections in the synthetic SZ maps, and a singleband filter for the X-ray detections. We identified halos as $S / N$ peaks in the filtered images and compared them with the parent distribution of dark matter halos contained in the original cosmological simulation. 
We find that multi-band filtering considerably reduces the number of spurious SZ detections and increases the fraction of halos reliably identified in the light cones. For instance, $50 \%$ of the halos with masses $M \simeq 10^{14} M_{\odot} / h$ are found when using a multi-band filter, compared to $30 \%$ with the single-band filter. The mass sensitivity of the multi-band filter is approximately four times higher than for the single-band filter. As expected, the sensitivity is virtually independent of redshift.

The fraction of spurious detections in the X-ray maps is typically low, and almost constant (about $5 \%$ ) for a large range of $S / N$ ratios. On the whole, the X-ray catalogues are more complete than the SZ catalogues, even when multi-band SZ filters are used. However, the redshift distribution of the halos detected through their X-ray emission is strongly peaked at low redshifts, and only massive halos can be detected at high redshift. This emphasises the importance of using complementary methods.

Weak-lensing halos detected with the optimal filter proposed by Maturi et al. (2005) reach a similar mass sensitivity as SZ and (soft) X-ray detections at redshifts where the lensing efficiency is highest, but clusters can be detected up to high redshifts. The contamination of weak-lensing halo catalogues is comparable to the X-ray catalogues, while the SZ contamination is much lower. Weak-lensing halo catalogues are similarly complete as SZ catalogues. For more quantitative details, see Pace et al. (2007).

Catalogues of common SZ and X-ray detections show that their mass function closely follows that of the SZ detections (due to the mass sensitivity of the filter), while their redshift distribution is very similar to that of the X-ray detections because X-ray clusters can be observed more reliably at low and intermediate redshifts.

Finally, we have shown that the detections represent the simulated halos in a virtually unbiased manner (see lower panels in Fig. 17).

Acknowledgements. We are grateful to Mauro Roncarelli for helpful suggestions on the simulation of SZ and X-ray maps. We thank Stefano Borgani and Giuseppe Murante for providing the outputs of the hydrodynamic simulation used in this work. We also thank an anonymous referee whose comments helped to improve the paper. Computations have been performed using the IBM-SP5 at Cineca (Consorzio Interuniversitario del Nord-Est per il Calcolo Automatico), Bologna, with CPU time assigned under an INAF-CINECA grant. We acknowledge financial contributions from contracts ASI-INAF I/023/05/0, ASIINAF I/088/06/0 and INFN PD51. This work was supported by the Deutsche Forschungsgemeinschaft (DFG) under the grants BA 1369/5-1 and 1369/5-2 and through the Transregio Sonderforschungsbereich TR 33, as well as by the DAAD and CRUI through their Vigoni program.

\section{References}

Arnaud, K. A. 1996, ASPC, 101, 17

Bertin, E., \& Arnouts, S. 1996, A\&AS, 117, 393

Bonaldi, A., Tormen, G., Dolag, K., \& Moscardini, L. 2007, MNRAS, 378, 1248B

Borgani, S., Rosati, P., Tozzi, P., \& Norman, C. 1999, ApJ, 517, 40

Borgani, S., Murante, G., Springel, V., et al. 2004, MNRAS, 348, 1078

Carlstrom, J. E., Holder, G. P., \& Reese, E. D. 2002, ARA\&A, 40, 643
Clowe, D., De Lucia, G., \& King, L. 2004, MNRAS, 350, 1038

Comerford, J. M., Meneghetti, M., Bartelmann, M., \& Schirmer, M. 2006, ApJ, 642, 39

da Silva, A. C., Barbosa, D., Liddle, A. R., \& Thomas, P. A. 2000, MNRAS, 317,37

Diaferio, A., Borgani, S., Moscardini, L., et al. 2005, MNRAS, 356, 1477

Diego, J. M., Vielva, P., Martínez-González, E., Silk, J., \& Sanz, J. L. 2002, MNRAS, 336, 1351

Doran, M. 2005, JCAP, 10, 11

Eisenstein, D. J., Zehavi, I., Hogg, D. W., et al. 2005, ApJ, 633, 560 Ettori, S., Borgani, S., Moscardini, L., et al. 2004, MNRAS, 354, 111

Fang, W., \& Haiman, Z. 2007, PhRvD, 75, 043010

Finoguenov, A., Reiprich, T. H., \& Böhringer, H. 2001, A\&A, 368, 749

Finoguenov, A., Guzzo, L., Hasinger, G., et al. 2007, ApJS, 172, 182

Haardt, F., \& Madau, P. 1996, ApJ, 461, 20

Haehnelt, M. G., \& Tegmark, M. 1996, MNRAS, 279, 545

Hallman, E. J., O'Shea, B. W., Burns, J. O., et al. 2007, ApJ, 671, 27

Hashimoto, Y., Barcons, X., Böhringer, H., et al. 2004, A\&A, 417, 819

Hennawi, J. F., \& Spergel, D. N. 2005, ApJ, 624, 59

Herranz, D., Sanz, J. L., Barreiro, R. B., \& Martínez-González, E. 2002a, ApJ, 580,610

Herranz, D., Sanz, J. L., Hobson, M. P., et al. 2002b, MNRAS, 336, 1057

Hobson, M. P., \& McLachlan, C. 2003, MNRAS, 338, 765

Hoekstra, H., Mellier, Y., van Waerbeke, L., et al. 2006, ApJ, 647, 116

Hütsi, G. 2006, A\&A, 446, 43

Kazantzidis, S., Kravtsov, A. V., Zentner, A. R., et al. 2004, ApJ, 611, L73

King, I. 1962, AJ, 67, 471

Kitayama, T., \& Suto, Y. 1997, ApJ, 490, 557

Knox, L. 1995, Ph. Rv. D, 52, 4307

López-Caniego, M., Herranz, D., González-Nuevo, J., et al. 2006, MNRAS, 370, 2047

Maturi, M., Meneghetti, M., Bartelmann, M., Dolag, K., \& Moscardini, L. 2005, A\&A, 442, 851

Mazzotta, P., Rasia, E., Moscardini, L., \& Tormen, G. 2004, MNRAS, 354, 10

Melin, J.-B., Bartlett, J. G., \& Delabrouille, J. 2006, A\&A, 459, 341

Mewe, R., Kaastra, J. S., \& Liedahl, D. A. 1995, Legacy, 6, 16

Monaghan, J. J. 1992, ARA\&A, 30, 543

Monaghan, J. J., \& Lattanzio, J. C. 1985, A\&A, 149, 135

Murante, G., Arnaboldi, M., Gerhard, O., et al. 2004, ApJ, 607, L83

Pace, F., Maturi, M., Meneghetti, M., et al. 2007, A\&A, 471, 731

Pierpaoli, E., Anthoine, S., Huffenberger, K., \& Daubechies, I. 2005, MNRAS, 359, 261

Puchwein, E., Bartelmann, M., Dolag, K., \& Meneghetti, M. 2005, A\&A, 442, 405

Rasia, E., Mazzotta, P., Borgani, S., et al. 2005, ApJ, 618, L1

Roncarelli, M., Moscardini, L., Tozzi, P., et al. 2006a, MNRAS, 368, 74

Roncarelli, M., Ettori, S., Dolag, K., et al. 2006b, MNRAS, 373, 1339

Roncarelli, M., Moscardini, L., Borgani, S., \& Dolag, K. 2007, MNRAS, 378, 1259

Rosati, P., Tozzi, P., Ettori, S., et al. 2004, AJ, 127, 230

Schäfer, B. M., \& Bartelmann, M. 2006, ArXiv Astrophysics e-prints [arXiv: astro-ph/0602406]

Schäfer, B. M., Pfrommer, C., Hell, R. M., \& Bartelmann, M. 2006, MNRAS, 370,1713

Sefusatti, E., Vale, C., Kadota, K., \& Frieman, J. 2007, ApJ, 658, 669

Sehgal, N., Trac, H., Huffenberger, K., \& Bode, P. 2007, ApJ, 664, 149S

Spergel, D. N., Bean, R., Doré, O., et al. 2007, ApJS, 170, 377

Springel, V. 2005, MNRAS, 364, 1105

Springel, V., \& Hernquist, L. 2003, MNRAS, 339, 289

Tang, J. Y., \& Fan, Z. H. 2005, ApJ, 635, 60

Vale, C., \& White, M. 2006, New Astron., 11, 207

Vikhlinin, A., McNamara, B. R., Forman, W., et al. 1998, ApJ, 502, 558

Weller, J., \& Battye, R. A. 2003, New Astron. Rev., 47, 775 